Bon droit et raison d'État. Réflexions sur les rapports entre le pouvoir royal et la justice du Parlement dans le dernier tiers du XIV ${ }^{\mathrm{e}}$ siècle

Michelle Bubenicek

\author{
(2) OpenEdition \\ Journals \\ Édition électronique \\ URL : https://journals.openedition.org/crm/891 \\ DOI : $10.4000 / \mathrm{crm} .891$ \\ ISSN : 1955-2424 \\ Éditeur \\ Honoré Champion \\ Édition imprimée \\ Date de publication : 15 février 2000 \\ ISSN : 1272-9752
}

Référence électronique

Michelle Bubenicek, «Bon droit et raison d'État. Réflexions sur les rapports entre le pouvoir royal et la justice du Parlement dans le dernier tiers du XIVe siècle », Cahiers de recherches médiévales [En ligne], 7 | 2000, mis en ligne le 03 janvier 2007, consulté le 15 décembre 2022. URL : http:// journals.openedition.org/crm/891; DOI : https://doi.org/10.4000/crm.891

Ce document a été généré automatiquement le 15 décembre 2022.

Tous droits réservés 


\title{
Bon droit et raison d'État. Réflexions sur les rapports entre le pouvoir royal et la justice du Parlement dans le dernier tiers du $\mathrm{XIV}^{\mathrm{e}}$ siècle
}

\author{
Michelle Bubenicek
}

1 Dans le cadre d'un État souverain, la nature des rapports existant entre la justice d'État et les intérêts de l'appareil gouvernemental a de tout temps excité la curiosité. En effet, il est loisible de se demander si l'État, source de justice, la rend toujours de façon impartiale ou si dans certains cas précis, notamment lorsque la «raison d'État» est en jeu, le cours des jugements peut être dévié de manière à contenter ou, du moins, à ne pas heurter de plus puissants intérêts.

2 À la fin du Moyen Âge, en particulier, au moment où se met en place l'État moderne, est-il aisé ou tout simplement concevable pour un justiciable, quel que soit son rang, d'obtenir satisfaction de la plus haute juridiction du royaume quand ses intérêts vont contre ceux du détenteur du pouvoir souverain?

3 Il semble intéressant de tenter de répondre à cette question à la lumière du contexte politique particulier de la seconde moitié du XIV e siècle, qui voit une succession de gouvernements aux pratiques opposées : au règne fort de Charles $\mathrm{V}$ succède, en effet, la minorité de son fils Charles VI et, si ce dernier reprend en main le pouvoir en 1388, les débuts de sa longue maladie remettent en cause son autorité dès 1392 ; la minorité royale, puis la maladie du roi permettent alors à ses oncles, comme chacun sait, de se partager le gouvernement pour en mieux profiter.

4 Dans ce contexte privilégié de «valse » des gouvernements, la démarche consistant à mettre en rapport les étapes du déroulement de longs procès à forte teinture politique avec les fluctuations de la raison d'État, dont le contenu est évidemment lié à la personnalité du détenteur du pouvoir suprême, permettrait de toute évidence de 
répondre en partie à la question de départ, celle de la partialité éventuelle de la justice royale dans des causes politiques.

5 C'est cette méthode que nous nous proposons à présent d'appliquer pour scruter le fonctionnement de la justice royale dans le dernier tiers du XIV ${ }^{e}$ siècle. Le matériau nous est fourni par deux longs procès en Parlement débutant, pour l'un, au début du règne de Charles $\mathrm{V}$ et, pour l'autre, au moment de l'arrivée au gouvernement de Charles VI des conseillers du roi défunt, les «Marmousets». Dans les deux cas, la partie lésée est une dame de haut rang, proche des cercles du pouvoir, Yolande de Flandre, comtesse de Bar et dame de Cassel (1326-1395). Et l'adversaire n'est autre que, dans le premier cas, Bertrand du Guesclin, favori de Charles V et, dans le second, Philippe le Hardi, frère de Charles V, duc de Bourgogne et comte de Flandre.

L'adversaire : Bertrand du Guesclin (1367-1385)Un procès pour douaire

6 Aux origines du procès qui opposa Yolande de Flandre à Bertand du Guesclin, on trouve la terre de Longueville, propriété du second mari de la comtesse de Bar. Philippe de Navarre - qui n'était autre que le frère du célèbre Charles le Mauvais, roi de Navarre avait en effet épousé Yolande de Flandre en 1353, au plus fort du conflit opposant le roi de France au trop puissant «clan navarrais». Cette alliance politique était venue fort à propos conforter les possessions territoriales de la famille de Navarre dans le contexte précis de la "guerre des mariages "1. Capitaine de guerre efficace pour son frère Charles, Philippe de Navarre mourut en 1363, laissant derrière lui les châtellenies d'Anet $^{2}$ et de Nogent-le-roi ${ }^{3}$, ainsi que le comté de Longueville ${ }^{4}$, qui embrassait notamment la riche ville de Montivilliers 5 . À titre de douaire, Yolande de Flandre réclama donc dès cette époque, comme la coutume l'y autorisait, la moitié des châtellenies d'Anet et de Nogent-le-Roi, ainsi que le tiers du comté de Longueville.

7 Malheureusement pour la comtesse de Bar, les biens de Philippe de Navarre avaient fait l'objet, dès 1363 (date de la mort de l'intéressé), d'une confiscation en bonne et due forme de la part de la couronne: trahison de Charles le Mauvais oblige ${ }^{7}$, le comté de Longueville, Anet et Nogent-le-Roi, biens du frère du Mauvais, furent donc saisis et réunis au domaine. Puis la victoire de $\mathrm{Cocherel}^{8}$, dont le principal artisan fut Bertrand du Guesclin, eut pour conséquence de faire donner le comté de Longueville au responsable du triomphe : le 27 mai 1364, Du Guesclin se vit donc remettre le comté, en toute propriété, héréditairement et sous la seule réserve de l'hommage; pour l'occasion, le chef de guerre obtint d'ailleurs la dignité comtale9.

Dans ce contexte exceptionnel - le règlement d'une trahison politique et des biens échus soit aux mains du roi lui-même (Anet, Nogent-le-Roi), soit aux mains d'un favori du roi (comté de Longueville) - il est significatif que Yolande de Flandre ait finalement concentré ses prétentions au douaire sur le comté de Longueville. Rompue aux pratiques du pouvoir, elle comprit, en effet, selon toute apparence, que le domaine du roi était en quelque sorte un domaine interdit et qu'elle n'avait de chances d'obtenir une partie de son dû qu'en s'adressant à une tierce personne, en l'occurrence Bertrand du Guesclin...

9 Charles $\mathrm{V}$ donna du reste son accord à cette revendication recentrée dans un acte du 17 juillet $1364^{10}$ et, plus encore, dans des lettres du 17 août $1367^{11}$, dans lesquelles, prévoyant l'opposition de son favori à donner satisfaction à la comtesse, il autorisait les parties à procéder : 
Et se ledit conte, ses genz [...] ou autres se wellent opposer au contraire, pour ce que c'est cause de doaire en lequele il appartient proceder somierement et de plain, donne certain jour [...] aus opposans [...] pour proceder et aller avant sur les choses dessus dites....

11 Cette citation donne le ton du procès qui va suivre : le roi est tiraillé entre son désir de maintenir son favori en possession du don royal qu'il lui a fait et l'obligation qui est la sienne, en vertu du serment du sacre, de rendre la justice de façon équitable, en faveur notamment des plus faibles, dont font partie les veuves comme Yolande.

12 Le procès qui débute en 1367 voit donc s'affronter en quelque sorte la raison d'État en la personne de Du Guesclin, artisan de la victoire française, bras droit du roi contre les Anglais, et le bon droit en la personne de Yolande de Flandre dont les prétentions à un douaire sont reconnues par la coutume en vertu de ce que l'on pourrait qualifier d'un «droit des veuves».

Douaire contre don royal

13 En effet, loin de chercher à trouver une autre récompense pour les bons et loyaux services de son chef de guerre, Charles $\mathrm{V}$ ne modifia en aucune manière la donation du comté de Longueville à la faveur d'un arrangement qui eût pu contenter Yolande ; au contraire, il s'efforça selon toute apparence de la rendre irrévocable, puisqu'au traité de Pampelune du 6 mars 1365, il échangeait officiellement avec le roi de Navarre Mantes, Meulan et le comté de Longueville contre la baronnie de Montpellier ${ }^{12}$, en vue de régler toute contestation ultérieure. Or, si Yolande se réclama plus tard, nous le verrons, d'un «droit des veuves » reconnu, elle ne pouvait sur le principe même de la donation s'en prendre au souverain qui était également, ce faisant, dans son «bon droit » le plus strict, celui consacré par l'adage bien connu «le roi peut donner la chose d'autrui».

14 Car le XIV siècle voit l'extraordinaire développement de la pratique des donations royales, en matière notamment de biens confisqués ${ }^{13}$ : effet du don royal, le donataire se trouve immédiatement saisi de sa nouvelle propriété, sans prescription, ce qui le met à l'abri de toute revendication, y compris celle de la partie lésée. Le second effet du don était toutefois d'ouvrir à cette dernière un recours contre l'auteur de la donation, en l'occurrence le roi, ce qui explique sans doute que Yolande se soit adressée à Charles V, dans l'été de 1367, dans l'espoir d'obtenir son dû. Mais on devine que l'opération était délicate...

15 C'est dans ce contexte précis que la comtesse eut à faire valoir son bon droit de veuve en attente de douaire. Car si le roi peut donner la chose d'autrui, il est admis qu'il lui faut tout de même veiller à sauvegarder l'équilibre des relations privées et à conserver, en cas de confiscation, les droits du conjoint du condamné, en particulier pour les questions de douaire ${ }^{14}$. Il ne pouvait, de plus, oublier qu'il se devait de protéger les miserabiles persone. Le Grand Coutumier de France, quant à lui, affirmait d'ailleurs nettement que les droits de la douairière étaient opposables au confisquant ${ }^{15} \ldots \mathrm{Et}$, de façon générale, on s'accordait à reconnaître que la trahison du mari, si haute fût-elle, ne devait pas empêcher sa veuve d'obtenir son douaire ${ }^{16}$.

Le procès de 1367 à 1385 : bon droit contre raison d'État?

$16 \mathrm{Au}$ vu de ces divers éléments, il est incontestable que le procès qui s'ouvrit à la fin de l'année 1367, opposant Yolande de Flandre à Bertrand du Guesclin, doit être analysé sous l'angle de la raison d'État: si le contexte de guerre (trahison navarraise, dévouement de Du Guesclin) a, en effet, motivé la confiscation du comté de Longueville 
et son octroi au capitaine breton à l'origine de l'affaire, ce contexte reste très sensible au cours du procès. Par ailleurs, ce don, manifestation d'une souveraineté pleine et entière chère au nouveau roi, ne pouvait pour cette raison même être révoqué ni même corrigé. Destiné à récompenser des services rendus, il devait enfin établir de manière définitive l'adhésion de Du Guesclin au camp royal et toute remise en cause était de ce fait inconcevable.

Il résulte de tout cela que la position de Du Guesclin est dès 1367 exceptionnellement confortable, situation perceptible dans le déroulement du procès en Parlement: si Yolande de Flandre a le droit pour elle, il est sensible que son adversaire, au-delà du chef de guerre, n'est autre que le roi ; aussi la cause de la comtesse apparaît-elle de toute évidence une cause perdue d'avance: lorsque des interêts de cet ordre sont en jeu, même le bon droit reconnu a du mal à s'imposer.

Ainsi, il est frappant de constater que jusqu'à la mort de Charles $\mathrm{V}$ et de son favori, survenues toutes deux au cours de l'année 1380, la cause de Yolande de Flandre en Parlement ne progresse aucunement. Et il faudra attendre la mort du connétable pour que l'affaire prenne un autre tournant: une fois disparu le héros de Cocherel et d'autres batailles mémorables, le bon droit peut enfin triompher; dès 1385, Yolande de Flandre parvient à un accord satisfaisant avec les héritiers et ayants-droit de Bertrand qui met un terme définitif à l'affaire.

Le déroulement du procès dans sa première phase $\mathrm{e}^{17}$, de 1367 à $1380^{18}$ est donc tout à fait instructif pour examiner la façon dont la justice royale procède pour repousser l'issue d'une affaire aux conséquences politiques gênantes.

La condition de «chargé de mission royal» de Bertrand du Guesclin semble d'ores et déjà avoir été l'un des facteurs principaux de l'hésitation persistante des juges royaux à traiter l'affaire. En effet, dès le début du procès, Bertrand du Guesclin, par procureur interposé, ne cesse de mettre en avant son statut exceptionnel d'envoyé en mission et de bras droit de Charles V: l'extrait du registre des plaidoiries ${ }^{19}$, qui nous permet de suivre le débat oral par lequel s'ouvrit la cause, nous relate par le menu le fait que Bertrand ne pouvait à l'époque régler l'affaire, étant donné sa présence en Espagne, sur ordre du roi, pour débarrasser le royaume des compagnies :

Bertran dit que l'entreprise qu'il commença fu du commandement du roy et pour l'utilité publique, pour widier le royaume des compaignez et pour guerroier les

Sarrazins quelque moien qui soit entervenu...

21 La formulation employée par le procureur de Bertrand du Guesclin ne laisse planer aucun doute sur la nature de la tâche qui lui a été confiée : il s'agit bien d'une mission d'État, puisque l'ordre émane du souverain en personne et que l'expression «pour l'utilité publique» insiste sur la notion de l'intérêt supérieur de l'État monarchique naissant. L'arrêt du conseil rendu le 20 novembre $1367^{20}$ rend d'ailleurs également compte de cet aspect des choses :

Dictus Bertandus diu et pro evidenti utilitate rei publice se absentaverat fueratque et erat propter certa impedimenta que sibi postea supervenerant in certa guerra captus et prisionarius...

22 Étant donné la date de l'arrêt, il est aisé de déterminer les événements auxquels les documents font allusion : il s'agit de l'expédition de Castille qui, sous la direction de $\mathrm{Du}$ Guesclin, devait permettre de libérer le royaume des compagnies désoœuvrées par la fin provisoire des hostilités contre l'Angleterre. Pour remédier au problème, Charles $\mathrm{V}$ 
avait, dès le mois de juin 1365, chargé Arnaud de Cervole de mener une partie des troupes guerroyer contre les Turcs, en suivant le Danube ${ }^{21}$. En 1366, le reste des compagnies devait être acheminé en Espagne, sous la conduite de Bertrand du Guesclin. officiellement, on justifia l'expédition par la volonté de lutter contre Pierre le Cruel, roi de Castille, tyran que l'on disait allié des Maures de Grenade ; en réalité, on s'attaquait à lui essentiellement parce qu'il était l'allié du roi d'Angleterre...22

La formulation utilisée par Bertrand du Guesclin a donc à juste titre d'incontestables accents de propagande et l'argument devait être entendu des tribunaux royaux: comme son maître Charles $\mathrm{V}$, le chef de guerre manie avec habileté les concepts de guerre sainte (guerroier les Sarrazins), de défense du royaume et du bien public (pour l'utilité publique / pro evidenti utilitate rei publice). L'affirmation qui suit prend dès lors toute sa valeur: au cours de cette mission d'État, Bertrand a été fait prisonnier; il lui est donc impossible de régler son litige avec Yolande : «or est prisonnier et si est sa partie plus favorable que la dame $»^{23}$.

À la bataille de Najera, en avril 1367, Bertrand du Guesclin fut, en effet, capturé par un aventurier anglais auquel il fut racheté, le 20 juillet de la même année, pour le compte du roi Edouard III $^{24}$. Une énorme rançon fut exigée : elle se montait à 100000 doubles de Castille. Le gouvernement royal, toutefois, ne la contesta pas et s'attacha à rassembler la somme, preuve s'il en est de l'importance acquise par Du Guesclin auprès du roi et au sein des milieux royaux ${ }^{25}$ : une rançon de cette importance ne pouvait qu'être le signe irréfutable de l'ascension fulgurante du chef de guerre. Dès la fin de novembre 1367, en tout cas, on négociait la libération de Du Guesclin, tractations contemporaines des débuts de la procédure entamée en Parlement par Yolande de Flandre.

Ces précisions diverses aident à mieux comprendre le contexte des réclamations de Yolande : il va sans dire qu'elles tombaient très mal. Que pesait, en effet, un douaire (un second, du reste, car pour Yolande de Flandre il s'agissait d'un remariage et Bertrand $\mathrm{du}$ Guesclin ne manquera pas de faire usage de l'argument) face au règlement d'une affaire politique de premier ordre: libérer le bras droit de la monarchie, devenu prisonnier lors de l'accomplissement d'une mission d'État?

Du Guesclin, du reste, on l'aura compris, ne se prive pas d'exploiter ce contexte très favorable: loin de se contenter de premières lettres d'état qui, on le sait, étaient accordées, au XIV ${ }^{\mathrm{e}}$ siècle, aux prisonniers et aux otages, à ceux que le service du roi appelait en mission ou encore à ceux qui, comme lui, allaient combattre contre les ennemis du royaume et de la Chrétienté, en ayant pour effet de suspendre les procédures engagées contre eux ${ }^{26}$, il en exigeait de nouvelles, dans le but évident de retarder le plus possible la procédure. ${ }^{27}$

Si la cour ne s'y trompe pas en repoussant la demande de Du Guesclin de se voir accorder de nouvelles lettres d'état (arrêt du Conseil du 20 novembre $1367^{28}$ ), ce qui confirme la tendance jurisprudentielle en vigueur depuis le début du siècle ${ }^{29}$, il n'en demeure pas moins que la suite des événements n'est pas pour autant favorable à Yolande de Flandre. En effet, le 23 décembre de la même année, un autre arrêt du Conseil ${ }^{30}$ était rendu dont le contenu très procédurier ne permettait aucune réelle avancée. S'il est manifeste que la cour y retint l'essentiel des arguments des avaocats de la comtesse, tandis qu'elle ne conservait de celles pour Bertrand du Guesclin que les plus acceptables - et pour cause ${ }^{31}-$, on constate cependant que la marche ultérieure de 
la justice, entre 1370 et 1380, date de la mort de Bertrand, est inexistante et qu'il faut attendre l'année 1382 pour voir se poursuivre l'affaire...

Ce premier intervalle de douze années environ, pour l'obtention d'un douaire qui aurait dû normalement l'être aux alentours de $1363^{32}$, mérite assurément d'être constaté afin de souligner la parallélisme troublant existant entre cet intervalle et la dernière phase d'ascension, à tous égards remarquable, de Bertrand du Guesclin : en octobre 1370, Charles V lui confiait la très haute charge de connétable; quelques dix années plus tard, le chef de guerre eut l'insigne honneur d'être enterré dans la nécropole royale de Saint-Denis ${ }^{33}$, jalons d'une gloire au service de l'État.

Si le douaire était un droit dont la veuve ne pouvait assurément être déboutée, l'obtention même de ce droit mettait souvent, on le sait, des années ${ }^{34}$. Toutefois, dans le cas spécifique de Yolande de Flandre, opposée pour l'occasion à un très grand favori de la couronne, ce délai semble avoir été, dans un premier temps, pour raison d'État, particulièrement long: selon toute apparence, parce qu'il aurait eu pour conséquence d'entamer les biens du plus proche conseiller de Charles $\mathrm{V}$, les juges royaux ne purent envisager alors le règlement de l'affaire...

Aussitôt après la mort du connétable, bientôt suivie par celle de Charles $\mathrm{V}^{35}$, la comtesse de Bar parait, du reste, avoir subitement retrouvé l'espoir de se voir rendre justice, car elle adresse dès 1381 à Charles VI une nouvelle requête ${ }^{36}$ où elle réclame de façon tout à fait significative la totalité de son douaire et non plus seulement le tiers du comté de Longueville $^{37}$. Une fois disparus l'auteur et le bénéficiaire de la donation du comté, autrement dit la raison d'État, elle put enfin voir triompher son «bon droit ».

L'adversaire : Philippe le Hardi (1388-1395)Aux origines de l'affaire : un apanage contesté

31 Parce-qu'elle était l'héritière, par son père, de la terre de Cassel en Flandre maritime, apanage qui constituait une enclave dans le comté de Flandre, la comtesse de Bar eut également fort à faire avec Philippe le Hardi, duc de Bourgogne, à partir du moment où ce dernier devint comte de Flandre ${ }^{38}$.

L'apanage de Cassel avait été constitué en 1320 par le comte Robert de Béthune au profit de son fils cadet Robert, père de Yolande, compensation qui devait provisoirement permettre de maintenir la succession de Flandre dans la branche aînée et d'éviter que la branche cadette ne s'en empare ${ }^{39}$. Très vite, cependant, cette concession territoriale, avantageuse, est mal vécue par les comtes de Flandre successifs: Louis de Nevers (1322-1346), puis Louis de Mâle (1346-1384) s'acharnent ainsi à grignoter l'assiette de l'apanage sans trop encore, cependant, empiéter sur les droits du seigneur apanagiste. Pas que le nouveau comte Philippe le Hardi s'empresse de franchir dès 1388. L'on peut dès lors parler d'une politique avouée de "réduction " de l'apanage, qui s'inspire d'ailleurs des méthodes utilisées parallèlement par la royauté à l'égard des apanages princiers, ce qui n'est pas étonnant concernant Philippe le Hardi, prince du sang et membre du gouvernement royal.

Pour Yolande de Flandre, ces empiétements répétés ${ }^{40}$ deviennent toutefois rapidement intolérables et motivent son appel à la justice royale dès le mois d'octobre 1388. Comme dans l'affaire du douaire de Longueville, l'appel au Parlement est l'ultime recours, l'ultima ratio, et la comtesse ne s'y résout qu'après avoir vu échouer toutes ses tentatives de conciliation à l'amiable ${ }^{41}$. Quand la pression devient trop forte, elle ne peut plus envisager que cette solution, si douteuse en soit l'issue. Il faut sans doute 
nuancer ce recours à la justice royale, sensible dans maintes affaires, car il semble que le but premier de la démarche ait été encore d'éviter le procès en amenant la partie adverse à composer; c'est d'ailleurs, apparemment, la crainte de voir l'affaire portée devant le Parlement, phénomène mal vécu par les populations flamandes, qui semble avoir poussé Philippe le Hardi à tenter un compromis, entre 1388 et $1389 . .$.

Aussitôt après la mort de Louis de Mâle, Yolande de Flandre avait pourtant espéré trouver auprès de Philippe le Hardi un appui pour recouvrer nombre de droits " croqués » par le défunt comte dans son apanage de Cassel. En 1385, en toute confiance, elle présentait à cet effet au duc une longue liste de doléances tout en flattant au passage l'esprit de justice de celui qui apparaissait alors, il ne faut pas l'oublier, comme le maître du royaume. Immédiatement après la mort de Charles $V$, la France est, en effet, régie par les oncles du jeune Charles VI ${ }^{42}$, mais c'est à Philippe le Hardi que revient principalement le pouvoir ${ }^{43}$, jusqu'à ce que le roi parvienne, en 1388, à s'émanciper de cette tutelle trop lourde. Une formule employée par Yolande en novembre 1388 est d'ailleurs tout à fait révélatrice de la façon dont les contemporains appréciaient l'influence réelle de Philippe le Hardi: "pour la poissance le duc de Bourgoigne, lequel avoit et a le gouvernement du royaume $»^{44}$. conséquence de provoquer l'appel de Yolande au Parlement le 12 du même mois ${ }^{45}$, celles-ci se montrent paradoxalement curieusement empressées à négocier au début du mois suivant : c'est que, dans le royaume, les équipes gouvernementales viennent de changer. À la fin du mois d'octobre 1388, le jeune Charles VI a secoué la lourde tutelle exercée par ses oncles et s'est montré désireux de gouverner seul. À cet effet, il a rappelé sans attendre les conseillers de son père, des hommes d'expérience que leurs rivaux nommèrent par dérision les Marmousets. Provisoirement chassé du pouvoir, Philippe le Hardi n'est donc apparemment plus certain de voir l'affaire réglée à son profit, aussi se hâte-t-il d'éviter la confrontation avec les tribunaux royaux. Cet empressement se comprend d'autant mieux que Yolande de Flandre aurait selon toute apparence également envisagé de demander au roi de «mettre en sa main » l'apanage de Cassel ${ }^{46}$, procédé qui, on s'en doute, ne devait pas réjouir outre mesure le duc de Bourgogne...

38 La saisie brutale par le duc, au sein de l'apanage de Yolande, des forteresses de Rode et de Bornem, au mois d'août 1389, voit cependant échouer le processus de conciliation et la procédure en Parlement est initiée par une enquête dès le mois de novembre $1389^{47}$. Après une année de procédure, on aboutit, le $1^{\text {er }}$ février 1391, à un ajournement du duc de Bourgogne devant le Parlement dont le motif était la détention des terres de Rode et de Bornem contre la volonté de Yolande de Flandre ${ }^{48}$. La comtesse de Bar semblait donc avoir des chances de se voir rendre justice, mais le 4 février de la même année, un arrêt 
interlocutoire du Parlement ${ }^{49}$ statuait, quant à lui, sur la question des empiétements perpétrés sur les terres de l'apanage de Yolande par les agents de Philippe le Hardi de façon assez décevante: afin d'obtenir plus ample satisfaction, Yolande de Flandre devrait faire la déclaration des possessions sur lesquelles avaient porté les empiétements ducaux, décision qui, comme nous le montrent les registres du conseil ${ }^{50}$, allait dans le sens du duc de Bourgogne avant tout désireux de gagner du temps ${ }^{51}$.

Et si Yolande de Flandre, moins d'un mois plus tard, soit le 8 mars 1391, s'exécute en remettant au procureur du duc de Bourgogne la liste des localités litigieuses ${ }^{52}$, force est de constater que l'accomplissement de ce qui ne devait être qu'une simple formalité n'ouvre pas, pour autant, une période de résolution du conflit, car les années 1392 à 1395 (date du décès de Yolande de Flandre) voient une inertie quasi totale du Parlement et une montée parallèle des empiètements ducaux dans l'apanage de Cassel.

Faut-il le rappeler? L'année 1392 est celle-là même qui ouvre une étape décisive de l'histoire du royaume de France. Avec la folie de Charles VI, le gouvernement royal entre dans une nouvelle phase d'influence des princes du sang et Philippe le Hardi ne fut pas le dernier à profiter de cette occasion d'exercer le pouvoir. À cette même époque, la frappe de monnaies sur le modèle royal, dans un nouvel atelier ${ }^{53}$, doit d'ailleurs être interprétée, de sa part, non seulement comme le signe d'une audace sans pareille, mais surtout comme celui d'un évident retour au pouvoir. Le geste est révélateur d'une puissance retrouvée dont Yolande de Flandre et l'apanage de Cassel allaient bientôt faire les frais.

41 Tandis qu'à partir de 1392 Philippe le Hardi intensifie sa politique de réduction de l'apanage ${ }^{54}$, s'acharnant à anéantir tous les droits seigneuriaux que Yolande de Flandre pouvait y exercer, on ne peut que remarquer, en face, l'étrange silence des institutions royales et, en particulier, du Parlement, alors qu'une procédure était en cours, concernant Yolande, depuis 1389... Il est alors aisé de mettre en rapport ce silence avec le regain de l'activité des princes au gouvernement qui, comme chacun sait, s'est accompagné d'un noyautage certain des institutions et, notamment, du Parlement ${ }^{55}$. Et s'il est vrai que le comptage des nouvelles fidélités au sein de la cour suprême du royaume reste délicat à effectuer, la tendance au renouvellement y est tout de même sensible ${ }^{56}$. Pour les princes, une certaine épuration avait d'ailleurs son importance dans la mesure où plusieurs des compétences du Parlement dépassaient largement le cadre du judiciaire pour prendre une tournure politique évidente : si le Parlement n'avait pas stricto sensu à prendre des initiatives politiques, il pouvait, en effet, s'opposer aux décisions de roi et $\mathrm{du}$ conseil $^{57}$; il se considérait, en outre, comme la cour administrative par excellence et les liens entre la haute administration et le gouvernement étaient nombreux ${ }^{58}$.

42 Dans ce contexte politique exceptionnel, il n'est donc pas étonnant qu'à partir de 1392, le Parlement n'ait pas rendu, dans le procès de la comtesse de Bar contre le duc de Bourgogne, le moindre jugement. Quand les conseillers de Philippe le Hardi peuplaient conseil du roi et grandes institutions, on imagine mal, en effet, que ces mêmes instances aient pu adopter une position favorable à Yolande de Flandre et envisager de procéder dans un sens défavorable à l'un des détenteurs du pouvoir ${ }^{59}$.

43 À cet égard, l'affaire qui opposa Philippe le Hardi à Yolande de Flandre à propos de l'apanage de Cassel présente de troublantes analogies avec celle que nous avons évoquée en premier lieu, le procès pour douaire mettant en cause le favori de Charles V, Bertrand du Guesclin. Assurément, quand l'adversaire d'un justiciable, même de 
haut rang, est le détenteur du pouvoir suprême - le roi ou un prince du sang - ou l'un de ses favoris les plus proches, le silence se fait au sein des institutions royales et la cause défendue, si juste soit-elle, est en quelque sorte mise entre parenthèses. L'intéret principal de l'analyse de ces deux affaires réside donc assurément dans le fait qu'elle aura contribué à prouver, si besoin en était, que la résolution judiciaire des conflits ne peut, dans certains cas précis, se faire sans l'accord du politique.

\section{NOTES}

1.F. Autrand, Charles V, Paris, Fayard, 1995, pp. 118sq. La maison d'Evreux-Navarre travailla, dès la génération du père du Mauvais, Philippe d'Evreux, à étendre sa puissance en jouant sur deux tableaux, celui de l'accroissement territorial et celui des fidélités. L'alliance conclue avec Yolande de Flandre, comtesse de Bar et régente, à l'époque, du Barrois pour ses fils alors mineurs venait, d'une part, conforter les positions navarraises dans l'espace lorrain, d'autre part, certains biens de la comtesse, sis dans le Perche, prolongeaient les possessions navarraises en Evrecin, vers le comté d'Alençon et vers Etampes. Cf. Quand les femmes gouvernent. Robe, droit et politique dans la France du XIV siècle : l'exemple de Yolande de Flandre (1326-1395), th. de doctorat, Univ. de Paris I, déc. 1998, à paraître prochainement, p. 170sq.

2.Anet, Eure-et-Loir, arr. Dreux.

3.Nogent-le-roi, Eure-et-Loir, arr. Dreux, ch.l.c.

4.Longueville, Seine-Maritime, arr. Dieppe.

5.Montivilliers, Seine-Maritime, arr. Le Havre.

6.Archives départementales du Nord (désormais AD Nord), B 454, nº 9385 : supplique adressée au roi.

7.Voir P.-C.Timbal, «La confiscation dans le droit français des XIII ${ }^{e}$ et XIV ${ }^{\mathrm{e}} \mathrm{s} . »$, Revue historique de droit français et étranger (désormais RHD), 1943, p. 54.

8.16 mai 1364 : victoire de Du Guesclin sur l'armée navarraise du captal de Buch, victoire qui vient fort à propos conforter la position de Charles $\mathrm{V}$, sacré deux jours auparavant.

9.R. Delachenal, Histoire de Charles V, Paris, Picard, 1910, t. III, pp. 113-114.

10.AD Nord, B 417, $\mathrm{n}^{\circ} 9212$.

11.AD Nord, B 417, $\mathrm{n}^{\circ} 10042$.

12.R. Delachenal, op.cit., t. III, p.191.

13.F.-X. Leduc, «Le droit du roi à donner la chose d'autrui à la fin du XIVe s. », RHD, 1967, pp. 612-639.

14.P.-C. Timbal, art. cit., 1944, pp. 35-60 ; voir p. 47.

15.Ibid., p. 48.

16.Voir l'exemple significatif donné par J. Vincent, «Douaire préfix et quotité coutumière dans la jurisprudence du parlement de Paris au XIV ${ }^{\mathrm{e}}$ s.», RHD, 1970, pp. 568-616, et notament pp. 576-577 : en 1360, une certaine Pernelle dont le mari, allié 
des Anglais, avait été condamné pour crime de lèse-majesté, avait obtenu normalement son douaire du Parlement, alors que les biens de son époux avaient fait l'objet d'une confiscation.

17.C'est-à-dire jusqu'à la mort de Bertrand du Guesclin et avant la phase de négociations qui va suivre, aboutissant à l'accord final de 1385, déjà évoqué.

18.Le nombre des pièces importantes rassemblées pour l'étude de ce procès s'élève à neuf (deux arrêts, un jugé, quatre lettres et deux accords) ; à titre de comparaison, le procès pour douaire étudié par R. Jacob, La minorité de Robert VI de Wavrin. Questions de bail, garde et douaire d'après la jurisprudence du Parlement, Liège, 1978, comprenait quant à lui un ensemble de neuf arrêts.

19.Archives nationales, $\mathrm{X}^{1 \mathrm{a}} 1469$, fol. 251.

20.Archives nationales, $\mathrm{X}^{1 \mathrm{a}} 21$, fol. $234^{\mathrm{v}}$ et $237-238^{\mathrm{v}}$.

21.R. Delachenal, op. cit., t. III, p. 223.

22.Ibid., pp. 248-250.

23.Archives nationales, $\mathrm{X}^{1 \mathrm{a}} 1469$, fol. 251.

24.R. Delachenal, op.cit., p. 403 et p. 411.

25.Voir Ph. Contamine, «Bertrand du Guesclin, la gloire usurpée ?», L'Histoire, 1980, 20, pp. 44-53: l'auteur a calculé l'équivalent en poids d'or des trois rançons successives exigées pour la libération du chef de guerre au cours de sa carrière et a pu mettre en valeur une progression flagrante qui montre une ascension sociale et politique incontestable : 106 kg d'or exigés en 1360; 155 en 1364; 460 en 1367 après Najera! De même que ses mariages successifs, l'augmentation des rançons jalonne de toute évidence une réussite fulgurante: Yolande de Flandre s'attaquait à un adversaire de taille.

26.F. Aubert, Histoire du Parlement de Paris de l'origine à François I'r (1215-1515), Paris, 1894, t. II, p. 205.

27. À la date de l'acte, en effet, les premières lettres d'état obtenues étaient encore valables, puisqu'une fois accordées elles ne permettaient la reprise des instances suspendues qu'un mois après le retour du combattant ou du prisonnier : P.-C. Timbal, La guerre de cent ans vue à travers les registres du Parlement (1337-1369), Paris, CNRS, 1961, p. 305, 400 et 403 .

28.Document cité supra.

29.Les tribunaux royaux étaient, en effet, habitués à de nombreux abus concernant les lettres d'état, notamment lorsqu'il s'agissait de douaires : pour cette raison, Philippe VI de Valois les avait même supprimées en 1346. Un siècle plus tard, l'ordonnance d'avril 1454 fit d'ailleurs disparaître toutes celles qui servaient à différer des affaires "privilégiées », en particulier celles «ou il chet provision comme de douaires », voir F. Aubert, op. cit., p. 205. Bien avant l'ordonnance de 1454, le cas de Yolande de Flandre s'inscrit donc tout à fait dans cette catégorie.

30.Archives nationales, $\mathrm{X}^{1 \mathrm{a}} 21$, fol. $299^{\mathrm{v}}-300^{\mathrm{v}}$.

31.Face à la demande justifiée de Yolande, Bertrand ne pouvait que multiplier les exceptions destinées à retarder la procédure, telle la déclinatoire de juridiction, l'argument du pétitoire, etc.

32.L'obtention immédiate et sans difficultés d'un douaire étant, cependant, on le sait, une chose fort rare.

33.Ph. Contamine, op. cit., p. 50. 
34.M.-Th. Caron, « Mariage et mésalliance : la difficulté d'être femme dans la société nobiliaire à la fin du Moyen Âge ", La femme au Moyen Âge, colloque de Maubeuge, 1990, pp.315-325, voir p.317.

35.Bertand Du Guesclin est décédé le 13 juillet 1380 ; Charles V, le 16 septembre 1380. 36.AD Nord, B 18882, $\mathrm{n}^{\circ} 23106$.

37.Les terres d'Anet et de Nogent-le-Roi, confisquées purement et simplement par Charles V à la suite de la trahison de Charles le Mauvais et de son frère, ont assurément semblé à Yolande trop difficiles à réclamer au lendemain de la mort de son époux ; comme nous l'avons déjà signalé, elle concentra alors ses prétentions sur le comté de Longueville, Du Guesclin, si en faveur soit-il, n'étant pas le roi lui-même.

38.Soit après la mort de son beau-père, Louis de Mâle, en 1384.

39.La naissance de l'apanage de Cassel trouve ses origines en 1305, lorsque le comte de Flandre Robert de Béthune se préoccupe de régler sa succession. Il a un fils aîné Louis, de santé fragile, et un puîné, Robert, père de la future Yolande ; son souhait est de voir revenir le comté à Louis et ses héritiers, mais ce désir pose problème dans la mesure où la représentation n'est pas admise par la coutume de Flandre : en cas de décès prématuré (et prévisible) du fils aîné du comte, le comté reviendrait donc non au fils de ce dernier, prénommé également Louis, mais à Robert, l'oncle excluant le neveu. Pour que la représentation pût fonctionner, il fallut une renonciation en bonne et due forme de la part de Robert de Cassel. C'est la raison pour laquelle l'apanage de Cassel fut créé : il devait en effet permettre à Robert de renoncer à ses droits de succession au comté, voir M. Bubenicek, op. cit., p. 545sq.

40.Rétablissement systématique de privilèges judiciaires municipaux par le duc sans l'aval de Yolande, pourtant seigneur de la terre de Cassel; destitutions directes de membres de l'administration de la comtesse, etc. , empiétements qui avaient pour but évident de ruiner l'autorité de l'apanagiste aux yeux des populations locales.

41.Cf. par exemple la lettre adressée au chancelier du duc de Bourgogne le 31 juillet 1389 (AD Nord, B 18822, $\mathrm{n}^{\circ}$ 23177) où elle déclare avoir «plus chier [...] son droit par voye amiable».

42. Charles $\mathrm{V}$ avait décidé de confier la régence du royaume à l'aîné de ses frères, Louis d'Anjou, et la tutelle des enfants royaux aux ducs de Bourgogne et de Berry. Très rapidement cependant, le duc d'Anjou prend le pouvoir et ses frères n'entrent au conseil que pour y contrebalancer l'influence des anciens serviteurs de Charles V qui se trouvent rapidement éliminés.

43.Car Louis d'Anjou et Philippe le Hardi s'entendent rapidement pour expédier Jean de Berry en Languedoc. Louis d'Anjou, quant à lui, est vite attiré en Italie et en Provence par la succession des Angevins de Naples. La place est libre pour Philippe le Hardi.

44.AD Nord, B 482, $\mathrm{n}^{\circ} 11778$ bis.

45.Ibid., B 483, $\mathrm{n}^{\circ} 11817$.

46.Ibid., B 18822, $\mathrm{n}^{\circ} 23227$.

47.Archives nationales, $\mathrm{X}^{1 \mathrm{a}} 37$, fol. 184 : Charles VI ordonne à son prévôt de Montreuilsur-Mer d'enquêter sur le différend existant entre la dame de Cassel et le duc de Bourgogne, la première ayant appelé au Parlement.

48. Archives générales du royaume, Trésor de Flandre, $1^{\text {ere }}$ série, $n^{\circ} 963$.

49. Archives nationales, $\mathrm{X}^{1 \mathrm{a}} 38$, fol. $149-150^{\mathrm{v}}$.

50.Archives nationales, $\mathrm{X}^{1 \mathrm{a}} 1475$, fol. $330^{\mathrm{v}}-331$. 
51.Philippe le Hardi réclame une «veue» des terres de l'apanage de Cassel sur lesquelles porte, notamment, la question du renouvellement des échevinages, revendication évidemment destinée à gagner du temps, puisqu'en la matière les ordres émanaient directement de sa personne...

52.AD Nord, B 486, $n^{\circ} 18720$ : le document énumère soigneusement toutes les localités concernées par les «atentats » perpétrés par les agents ducaux et l'on constate avec intérêt que toutes les villes importantes de l'apanage y figurent, à savoir Dunkerque, Cassel, Warneton et Bourbourg.

53.M. Norberg, Les ducs et la royauté. Étude sur la rivalité des ducs d'Orléans et de Bourgogne (1392-1407), Stockholm, 1964 (Studia historica Upsalensia), p. 7.

54.Celle-ci trouve son aboutissement en janvier 1395, lorsque des troupes armées du duc de Bourgogne furent chargées de saisir Dunkerque, Hazebrouck, Nieppe et Cassel, tandis que les baillis d'Ypres et de Courtrai s'emparaient du château de Warneton. Bruxelles, Archives générales du royaume, CC, $n^{\circ} 14282$ et $n^{\circ} 14542$. Le prétexte était le refus de Yolande de Flandre de présenter au duc le dénombrement des terres de l'apanage de Cassel, ibid., n² 20285. Yolande, en effet, prétendait à juste titre qu'elle n'avait pas à faire le dénombrement de l'apanage quand aucun des prédécesseurs de Philippe le Hardi ne l'avait exigé d'elle-même ou de son père.

55.R. Vaughan, Philip the Bold. The formation of the Burgundian State, Londres, 1962, pp. 44-45.

56.M. Norberg, op. cit., p. 60, montre, en effet, que si une certaine prédominance bourguignonne au Parlement est une réalité de la fin du XIV ${ }^{\mathrm{e}}$ siècle, il est délicat d'en faire la démonstration rigoureuse dans la mesure où les choix des personnes se faisaient par cooptation : la démonstration reviendrait alors à faire la preuve d'une pression indirecte sur les membres en place, ce qui est malheureusement impossible. 57.Ibid., pp. 58-59.

58.Ibid., p. 58.

59.L'affaire prouve d'ailleurs, à l'inverse, que l'influence de Philippe le Hardi au sein du gouvernement central du royaume fut bien réelle et au moins égale à celle d'un Jean de Berry ou d'un duc d'Orléans, alors que l'on a généralement coutume d'insister sur la prédominance de ce dernier personnage à partir de 1393. Pour Jean de Berry, voir l'étude de R. Lacour, Le gouvernement de l'apanage de Jean, duc de Berry (1360-1416), Paris, 1934, pp. 308-309: l'auteur insiste bien sur le fait que les procès en Parlement contre le duc sont extrêmement difficiles à soutenir pour des particuliers, qui, la plupart du temps, se résignent à donner raison au duc. .. La conduite du Parlement est toujours très timide quand il s'agit de lui donner tort et, même dans des affaires graves, la pression est telle que la Cour s'efface devant la puissance ducale. Cf., par exemple, le cas d'une jeune fille enlevée et séquestrée par le duc qui voulait la marier selon son gré : ajourné devant le Parlement, le duc se fâche et écrit tout de suite au président du Parlement une lettre où il le menace de s'en prendre à sa personne et à ses biens si l'affaire se termine autrement qu'il en a décidé! L'affaire donne une petite idée de la liberté des institutions centrales de l'époque vis-à-vis des princes du sang, a fortiori de Philippe le Hardi. 


\section{AUTEUR}

MICHELLE BUBENICEK

Université de Besançon 